\title{
El impacto económico de las remesas en el ingreso de las familias mexicanas en la encrucijada del Covid-19
}

\section{The economic impact of remittances on the income of Mexican families at the crossroads of the Covid-19}

\author{
Renato Pintor-Sandoval y Jesús Bojórquez-Luque
}

Facultad de Estudios Internacionales y Políticas Públicas de la Universidad Autónoma de Sinaloa, México/Universidad Autónoma de Baja California Sur, México

\begin{abstract}
Resumen
El presente trabajo aborda la situación y el comportamiento que obtuvieron las remesas en el ingreso de las familias mexicanas durante la pandemia y sus efectos posteriores. Se entiende que las remesas han sido la salvaguarda de muchas comunidades y hogares en México, donde cerca de 1.65 millones reciben esa ayuda. La investigación hace un análisis de los índices de desempleo/empleo de la comunidad mexicana en los Estados Unidos; de las remesas recibidas hasta junio de 2020, así como los estados de la República Mexicana y su repercusión en sus hogares. Se concluye que, contrario a lo que podría pensarse derivado de la pandemia, las remesas se han elevado a partir de expresiones de solidaridad, de subsistencia familiar por la situación de riesgo sanitario, pero, sobre todo, al objetivo mismo de la remesa familiar, que la familia subsista y sea un componente básico para los que la reciben. La metodología que se emplea es cuantitativa, utilizándose información estadística de distintas fuentes, tanto de México, como de Estados Unidos, para un análisis desde distintos enfoques de las remesas, que nos permitirá observar el accionar de estos recursos.
\end{abstract}

Palabras clave: Remesas familiares, México, Pandemia.

\begin{abstract}
This paper addresses the situation and the behavior of remittances in the income of Mexican families during the pandemic and its subsequent effects. It is understood that remittances have been the safeguard of many communities and households in Mexico, where about 1.65 million receive such assistance. The research makes an analysis of the unemployment/employment rates of the Mexican community in the United States; of the remittances received up to June 2020, as well as the states of the Mexican Republic and their impact on their households. It is concluded that, contrary to what might be thought due to the pandemic, remittances have increased due to expressions of solidarity, family subsistence due to the health risk situation, but, above all, to the very objective of family remittances, that the family subsist and be a basic component for those who receive them. The methodology used is cuantitative, using statistical information from different sources, both from Mexico and the United States, for an analysis from different approaches to remittances, which will allow us to observe the actions of these resources.
\end{abstract}

Keywords: Family remittances, Mexico, Pandemic.

Artículo recibido el 24 de septiembre de 2020 y aprobado el 05 de febrero de 2021. 


\section{INTRODUCCIón}

$\mathrm{E}$

interés de este trabajo se suscribe en los acontecimientos que ha detonado la pandemia en las remesas familiares que los mexicanos envían a sus familias, enmarcado desde una perspectiva de dificultades económicas que atraviesa la situación del Covid-19. Sobre todo, nos interesa responder a las siguientes interrogantes: ¿Cómo se han visto afectadas o beneficiadas las familias remitentes ante un panorama nada halagador? ¿A qué se debe el incremento de las remesas en México?

En la actualidad, hay alrededor de 763 millones de migrantes internos en todo el mundo y 272 millones de migrantes internacionales, por lo que un poco más de mil millones de personas se encuentran en movimiento. De los cuales, casi 71 millones de personas se ven obligadas a abandonar sus hogares debido a conflictos armados, violencia generalizada o desastres naturales. De estos, casi 26 millones son refugiados; 41,3 millones son desplazados internos y 3.5 millones son solicitantes de asilo (OIM, 2020).

Para el caso de la migración mexicana, el Consejo Nacional de Población (CONAPO) (2017) comenta que ésta ha diversificado su destino en épocas recientes, donde la migración mexicana a Estados Unidos ha decrecido, como no se había observado desde hace una década (Zong, Batalova y Burrows, 2019). Sin embargo, es la Unión Americana, el principal destino que sigue concentrando la mayor parte de migrantes mexicanos, ya que según datos del Instituto de los Mexicanos en el Exterior (IME, 2018) registra que 11.913.989 connacionales radican en el exterior, 97.79 por ciento se ubican en Estados Unidos; mientras que cifras de la United States Census Bureau (USCB, 2018) estima que alrededor de 38.5 millones de personas son residentes en Estados Unidos de origen mexicano. En 2018, 12.3 millones son personas nacidas en México y 26.2 millones son mexicanos de segunda y tercera generación en Estados Unidos, es decir, son personas con uno o ambos padres nacidos en México y personas que se autodefinen como de ascendencia mexicana, respectivamente.

Entre otras cifras que es necesario mencionar, uno de cada dos mexicanos que reside en Estados Unidos, tiene entre 15 y 44 años de 
El impacto económico de las remesas en el ingreso de las familias ...

edad, ubicando la edad promedio de esta población en 24 años (IME, 2018). Una tercera parte (32.8 por ciento) de los inmigrantes mexicanos cuenta con ciudadanía, 19 por ciento llegó a esa nación entre 2006 y 2016 (IME, 2018).

Si bien se han modificado los patrones migratorios, entre el origen y el destino de los migrantes mexicanos, como comenta Armendares (2018) la mayoría aún procede de entidades migratorias como Michoacán (10.1 por ciento), Jalisco (9.4 por ciento) y Guanajuato (9.1 por ciento), entre otras, y que se asientan en California (31 por ciento), Texas (15.5) e Illinois (4.6 por ciento), pero que también, otras localidades se han sumado, dado el proceso migratorio mexicano (Chishti y Hipsman, 2015). En comparación, con otros grupos de migrantes en Estados Unidos, los mexicanos son más jóvenes (BBVA Research, 2018), tienen menos escolaridad y dominio del inglés, tienen menor recurso anual y en menor medida tienden a nacionalizarse (IME, 2018). A esto hay que agregarse a nuevas facetas del proceso migratorio, tales como el retorno, que entre 2005 y 2018 regresaron a México más migrantes de los que partieron a Estados Unidos, esto en parte a tres factores: como lo explica Cueto et al. (2019):

1. La crisis económica global del 2007-08 (Alarcón et al., 2009).

2. La aplicación de leyes migratorias antiinmigrantes tanto nacionales como estatales de Estados Unidos (Magaña, 2013).

3. Envejecimiento de la población mexicana de las principales regiones de expulsión (esto significa que los flujos de migración se invirtieron por primera vez en cerca de siete décadas) (OCDE, 2018).

Aún con estos indicadores de la migración mexicana señalados anteriormente y que podrían poner en entredicho el aporte de las remesas, el estudio de las remesas históricamente es y ha sido un tema central dentro de los estudios migratorios en México durante décadas, tales como los trabajos de Canales (2015); Díaz y Ramírez (2017); Carvajal y Almonte (2011). Estos trabajos, coinciden que estos recursos, comúnmente suelen compararse con algunos indicadores de manera macroeconómica, como una importante fuente de divisas y que superan los ingresos por exportaciones y los ingresos derivados de la Inversión Extranjera Directa (Calderón, 2015), incluso en el petrolero. Las remesas son significativas en el Producto Interno Bruto (PIB) de México; pero más allá de este indicador, las aportaciones que realizan los migrantes, son la principal fuente de subsistencia de algunas comu- 
nidades de escaso desarrollo regional. Se podría afirmar, incluso, que sin ellas muchas comunidades habrían desaparecido y sus habitantes emigrado hacia zonas más productivas (López et al., 2011). En cuanto al uso y aprovechamiento de las remesas en México, se abrió un debate, desde la década de los noventa, que hizo surgir dos escuelas principalmente, una la llamada "optimista" que visualizan las remesas, bajo el monto total, y al resultado que se podría esperar de ellas, por ejemplo, al compararlas con los ingresos de la Inversión Externa Directa (IED) o incluso el petróleo, como los trabajos de Arroyo y Berumen (2000); Montoya (2004) o Conway y Cohen (1988), trabajos que hablan sobre los bondades de estos recursos.

Por su parte, la escuela "menos resplandeciente" sobre dichos recursos, consideran que "están funcionando como un freno al desarrollo local, siendo que en gran parte generan una dependencia muy perniciosa en los hogares que las reciben: "Solo una pequeña proporción es orientada hacia las inversiones, como la mejora o la adquisición de una casa, o bien, el establecimiento de un pequeño negocio", como lo dice Binford (2003); Suro (2005), Canales (2015) o Carling (2007) donde este último considera que la magnitud y aporte de los ingresos que envían los migrantes constituyen "la centralidad que fueron adquiriendo las remesas en las agendas de las instituciones dominantes en el tema del desarrollo". Para éstas, se comenzó a gestar un viraje hacia el terreno "más optimista de los posibles efectos que podían tener las remesas", ese optimismo se fundamentaba en las nuevas concepciones de la división entre consumo e inversión (p. 58).

Sin embargo, al parecer resulta arriesgado sostener la importancia que dichos recursos funcionen como un detonador productivo y el eje fundamental del desarrollo económico, pues se olvida que gran parte de ellas se destinan al autoconsumo donde tienen sus efectos positivos, a nivel micro, por lo que su impacto se da en diferentes grados y efectos.

El objetivo de este artículo es entender el comportamiento que tendrá el dinero enviado por migrantes mexicanos vía remesas, bajo el impacto que se vislumbra en el desarrollo regional mexicano, ante la contingencia del Covid-19. Partimos de lo planteado por Castles (2016) que señala que durante la última década gran parte de los estudios entre migración y desarrollo, las remesas aparecen como instrumentos a correlacionar, por lo que se ha tornado un tema central en el debate académico y político a escala internacional, parte de esta 
discusión por los organismos internacionales, gobiernos de los países emisores y receptores de migrantes, centros de investigación, y otros sectores sociales, lejos de ayudar al estudio del desarrollo regional, no se antepone al migrante y a las necesidades adherentes a su entorno de bienestar (Robertson, 2018).

El trabajo se ha planteado de la siguiente forma, el primer apartado se toca a lo referente a la pandemia global del Covid-19 y los migrantes mexicanos en Estados Unidos, en la cual, los pronósticos no son nada alentadores, de acuerdo con Clemente et al. (2021) el Coronavirus que, a 13 meses y medio de su aparición, tiene una propagación global que alcanza a todos los países, enfermando a más de 107 millones, causado más de 2.3 millones de fallecimientos. Pondremos un especial interés de cómo el virus ha provocado la suspensión de actividades financieras, industriales, comerciales, sociales, culturales y deportivas, impactando en la economía estadounidense y, por consiguiente, en los migrantes mexicanos.

En el segundo apartado, se hablará de la correlación de las remesas familiares, no solo en la era del Covid-19, sino de un tiempo atrás, donde lejos de mitigarse su aportación a la economía mexicana, estas se han incrementado constantemente, como así lo hace ver González (2015) que, desde 2015, las remesas han ido en constante crecimiento, aún ante las amenazas de Trump de anteponer un arancel a las mismas (Pintor y Rocha, 2020) e incluso, ante la ola de deportaciones iniciados a partir de la administración de Clinton en la etapa de leyes estatales y federales antiinmigrantes en Estados Unidos (Pintor y Rocha, 2018).

Posteriormente daremos pie a nuestras conclusiones, recalcando que las remesas son parte importante, tanto para los indicadores macro económicos en México, ya que contribuyen en la economía de millones de familias que ven al norte como la tabla de salvamento en escenarios de depresión económica constante, y sobre todo en este escenario global, estos recursos hay que observarlos también en su aspecto micro social, ya que hay que considerar el desarrollo humano de quienes la reciben, en este escenario que se ha desenvuelto, debido por la pandemia, haciéndose de un mundo sumamente desigual y en constante transformación y vulnerabilidad económica.

\section{MigRaCión MEXICANA Y EL Covid-19}

El 31 de diciembre de 2019, la Comisión Municipal de Salud y Sanidad de Wuhan (provincia de Hubei, China), informó sobre un grupo de 27 
casos de neumonía de etiología desconocida, con una exposición común a un mercado mayorista de mariscos, pescado y animales vivos en la ciudad de Wuhan, incluyendo siete casos graves (Diario Farma, 2020). El inicio de los síntomas del primer caso fue el 8 de diciembre de 2019. El 7 de enero de 2020, las autoridades chinas identificaron como agente causante del brote un nuevo tipo de virus de la familia Coronaviridae que posteriormente ha sido denominado SARS-CoV-2, cuya secuencia genética fue compartida por las autoridades chinas el 12 de enero (Liu et al., 2020).

El día 11 de marzo, la Organización Mundial de la Salud (OMS) declaró la pandemia mundial. Desde el inicio de la epidemia a la fecha de este informe, se han detectado 31,181,172 casos en el mundo, más de 962 mil muertes, siendo países como India (5.4 millones); Brasil (4.56); Rusia (1.1); Colombia (0.77); Perú (0.76) y México (0.69) (Información hasta el 21 de septiembre de 2020, BBC, 2020), como los países con mayores índices de infectados.

Bajo esa tesitura, más allá de los problemas de la pandemia, también hace su aparición una nueva forma de guerra fría, donde no se trata meramente el interés ideológico como la que se vivió durante gran parte del siglo XX, sino al reacomodo mundial, no meramente militar, sino comercial, como lo ejemplifica, Loyer y Giblin (2020) donde mencionan que Estados Unidos parece desvanecerse como potencia frente a las ambiciones chinas, mientras que la debilidad europea es patente. El caso de España, Italia y Francia, sociedades seriamente tocadas por la crisis sanitaria, ejemplifican, el caos y la incertidumbre de lo que puede pasar. Donde el régimen autoritario chino y los Estados democráticos occidentales dependen de las consideraciones internas, principalmente en lo comercial y económico.

En su gran mayoría, la respuesta de los países es actuar bajo la instalación de la cuarentena, Borbón (2020, p. 57) comenta que:

La cuarentena es también una medida extrema que puede ser decretada por la autoridad, para obligar a la población a permanecer en sus casas y no salir de ellas hasta que se cumpla el plazo establecido, con el objetivo y único fin de frenar la propagación del virus.

De igual forma, el vivir en confinamiento domiciliario trae consigo nuevas formas sociales, clasistas y económicas, permitiendo el esclarecimiento de una higienización de la población, sobre esto Espinel (2020) afirma que el Covid-19, ha impactado de forma significativa, 
la manera como socialmente se ha materializado la geografía política de los cuidados, especialmente de las personas mayores. La forma de geografía política de los cuidados, institucionalmente hablando, ya se encontraba previamente debilitada, en varias partes del mundo, ante el desvanecimiento del Estado de bienestar social de varios países, que transitaron a nuevas formas de actividad productiva; entre ellos los servicios médicos a nivel global, he ahí, la instalación de la cuarentena.

Si bien, se han cerrado algunos de los negocios de forma global, el trance de la economía mundial ha sido alarmante, donde ni siquiera Estados Unidos, en un esfuerzo puramente nacional, puede superar la pandemia, donde la pérdida de empleos fue de 14.7 por ciento, cerca de 20.5 millones de empleos perdidos en abril de 2020, según la Oficina de Estadísticas Laborales (BLS, por sus siglas en inglés) (CNN en español, 2020), donde uno de cada cinco trabajadores ha hecho uso de la ayuda de desempleo (CNN en español, 2020). Aunque otras fuentes comentan que las cifras ascienden a 22 millones (Véase Figura 1) (Forbes, 2020a). A esto hay que sumarse, que esas pérdidas llegan después de fuertes recortes en marzo, cuando los empleadores recortaron 870 mil fuentes laborales. Esos dos meses equivalen a despidos tan severos que doblan los 8.7 millones de empleos perdidos durante la crisis financiera del 2007-08 (Alarcón et al., 2009).

Sin embargo, posterior a marzo, la economía norteamericana ha ido ganando poco a poco de lo perdido con anterioridad, por ejemplo, se crearon 2.5 millones de puestos de trabajo adicionales, poniendo fin aparente al proceso de destrucción de empleos que entre marzo y abril dejó en la calle a más de 21 millones de personas, sobre todo porque se han ido levantando las restricciones que se habían realizado, en las áreas de hostelería, construcción y servicios sanitarios, pero también en la educación y en los comercios al por menor.

De acuerdo con la BBC (2020) los negocios pequeños, son los que más se han beneficiado, siendo las contrataciones por parte de bares y restaurantes, que representaron casi la mitad de todos los empleos creados en julio, mientras que los consultorios odontológicos equivalen a diez por ciento. 





Es en Estados Unidos donde la pandemia ha cobrado mayores víctimas, sumando más de 6'852,213 casos confirmados; 3'724,373 curados y 199,633 fallecidos, siendo los estados de Nueva York, Nueva Jersey, Illinois, Massachusetts, California, Pensilvania y Michigan ${ }^{1}$ (americadigital, 2020), como lugares donde más han sufrido los padecimientos del Covid-19, y que en su gran mayoría pertenecen a la región noreste y del medio oeste, como se ilustra en el Mapa 1. Además, que estos estados, concentran casi 23.9 por ciento de la población emigrada mexicana en Estados Unidos, por lo que su riesgo a contagio y fallecimiento es letal. Mientras que la región de Estados Unidos que destacan es la del Sur (44.1 por ciento), el Oeste (24.8 por ciento), el Suroeste (17.2 por ciento), el Medio Oeste (5.7 por ciento), el Atlántico Medio (cinco por ciento) y Nueva Inglaterra (3.2 por ciento) (Moctezuma et al., 2018,)

Realizando un análisis, se antepone que la comunidad afroamericana, como la población más afectada por el Covid-19, le sigue la comunidad latina como las más vulnerable, por ejemplo, la ciudad de Nueva York, el epicentro de la pandemia en Estados Unidos, hasta el 8 de abril, el 28 por ciento de las 4,009 muertes por Covid-19 eran personas afro. En Chicago, la sexta ciudad más poblada del país, cerca de la mitad de los contagiados eran personas afrodescendientes, siguiendo de la población latina. En el estado de Michigan, este segmento poblacional está conformada solo por 14 por ciento, pero acumulan 33 por ciento de los casos reportados de Covid-19 y 41 por ciento de las muertes (Serrano, 2020).

Para el caso de la migración mexicana asentada en Estados Unidos, Valdivia (2020) nos dice que, debido a su situación precaria, el tipo de labores que realiza y su acceso a los servicios médicos, la población mexicana en Estados Unidos, resienten particularmente los efectos de la crisis sanitaria y la desaceleración económica que ocasiona el Covid-19. En cuanto a las mujeres, aumenta su vulnerabilidad, ya que se encuentran en mayor proporción en empleos de baja productividad (servicios) o bien fuera del mercado laboral (trabajo doméstico) (Manjarrez, 2020).






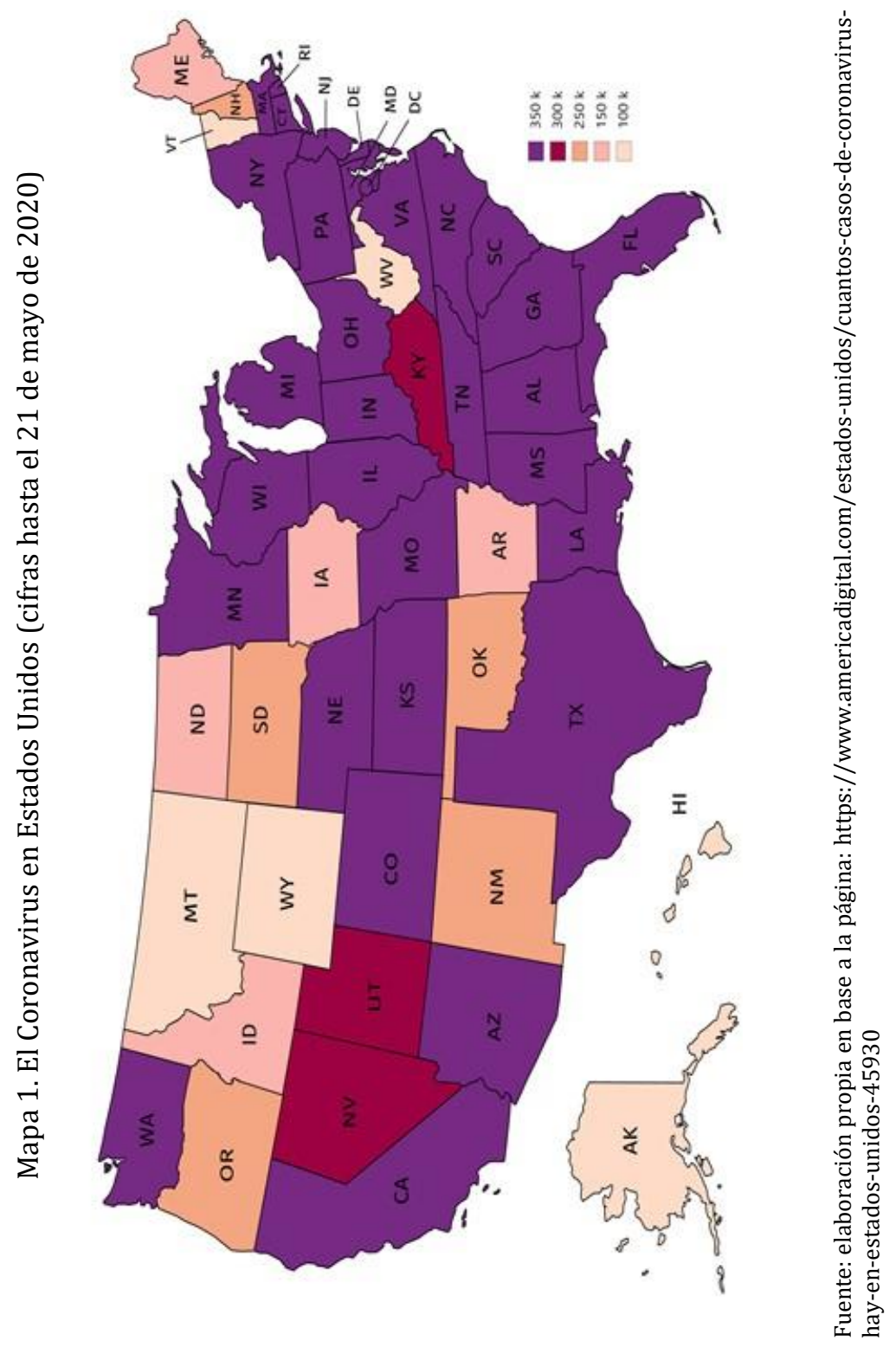


Dentro de los análisis estadísticos sobre los migrantes mexicanos en la Unión Americana, confirman esta tendencia: 86 por ciento de los mexicanos en Estados Unidos tiene menos de 62 años (USCB, 2018) lo cual teóricamente podría reducir el riesgo de complicaciones en caso de contagio de Covid-19. Asimismo, la Encuesta Nacional de Salud (NHIS, por sus siglas en inglés) muestra que la población hispana en ese país (60 por ciento, de la cual proviene de México), presentan menores tasas de asma y enfermedades cardíacas que la población no hispana. Sin embargo, presenta otros factores de riesgo como mayor prevalencia de obesidad (35 por ciento) y diabetes (proporción entre 13 y nueve por ciento) (Mazurek y Syamlad, 2018).

Además, a esto hay que sumarle la falta de cobertura médica. En 2018, 37 por ciento de los migrantes mexicanos no contaba con seguro médico, en comparación con nueve por ciento de la población estadounidense, lo que no sólo recrudece las consecuencias financieras de contagiarse, sino que reduce la posibilidad de procurar un tratamiento médico (Celorio, 2020). Bajo las circunstancias particulares de esta pandemia, se traduce en mayor riesgo de contagio para la comunidad migrante en su conjunto. Más aún, la precariedad de sus condiciones laborales (Valdivia 2020) y de vivienda hace prácticamente imposibles las medidas de distanciamiento social. En Estados Unidos, las poblaciones migrantes hispanas son tres veces más propensas a vivir en condiciones de hacinamiento (OCDE, 2018).

En cuanto al empleo, los migrantes mexicanos son más vulnerables al efecto de la recesión económica, dada su condición ilegal o indocumentada. De acuerdo a estimaciones del Pew Research Center (2017), la población mexicana sin documentos en Estados Unidos, representa 51 por ciento del total de quienes radican en ese país en esta condición, disminuyendo y manteniéndose estable en los últimos años, por lo que a medida que la pandemia progresa, la tasa de desempleo irá en aumento, ya que para el conjunto de los trabajadores en Estados Unidos, donde la tasa de desempleo hispano se agudizó de febrero a marzo de 2020, en 1.6 por ciento, comparado con 0.9 por ciento para la población en su conjunto.

Los datos anteponen que, en realidad en la comunidad latina, paso de 18.5 por ciento a 17.2, siendo el segmento racial que más recuperación tuvo en comparación a los afro estadounidenses, que tuvo un repunte de subir de 16.4 hasta 16.8 por ciento y en el de los asiáticos aumentó 0,5 por ciento hasta 15 por ciento (BBC, 2020). 
Además, es previsible que, con el deterioro de su salud física y las oportunidades laborales, disminuya su capacidad para enviar remesas a sus familiares, en su mayoría hogares de bajos ingresos, lo que a su vez afectará la economía mexicana. Las vulnerabilidades de los migrantes durante esta pandemia, por lo tanto, aumentarán en los hogares que dependen de ellos (Valdivia, 2020). Sobre todo, que, en 2016, se había registrado el índice de pobreza más bajo de la población mexicana en Estados Unidos, de pasar de 35.7 por ciento en 1994 de la población migrante mexicana a 19.8 por ciento en 2016 , por lo que los efectos de la pandemia podrían volver a los números de 30 o 40 por ciento de la población en situación de pobreza, como se ve en la Figura 2.

Esta figura denota, que sí bien, se habían bajado los índices de pobreza dentro de la comunidad emigrada mexicana, es en parte, a su tasa de desempleo que ha ido a la baja, donde también se representa por el poder adquisitivo, aunque con otros grupos étnicos, siguen siendo de los más bajos. En parte, la gran mejoría presentada por la comunidad mexicana emigrada en Estados Unidos, obedece al despunte que ha tenido nuevamente el mercado de la construcción, donde ocupa 16.4 por ciento del total empleado, siguiendo la hostelería y esparcimiento, con 14.1 por ciento; profesionales y administrativos, 13.6 por ciento; manufacturas, 12.5 por ciento; comercio 9.5 por ciento; Salud y educación 9.4 por ciento y la agricultura con el 5.4 por ciento, entre las principales actividades (USCB, 2018 y CONAPO, 2019). Precisamente, esas áreas son donde se han creado la mayor parte de empleos para el bimestre julio-agosto, donde la comunidad mexicana ha podido reinsertarse económicamente.

\section{LAS REMESAS EN MÉXICo EN LA ÉPOCA DEL CoVID-19}

En el trascurso de la cuarentena en México y Estados Unidos, han aparecido ciertas conductas, no muy apropiadas, que en algunos casos son ruines, como el acaparamiento de bienes de consumo, la especulación de los precios, la incertidumbre y el individualismo autoritario, como lo describe Salazar (2020: 203): "ubicó la pandemia a los más necesitados en una situación franca de dependencia", incluso el autor citado agrega: 


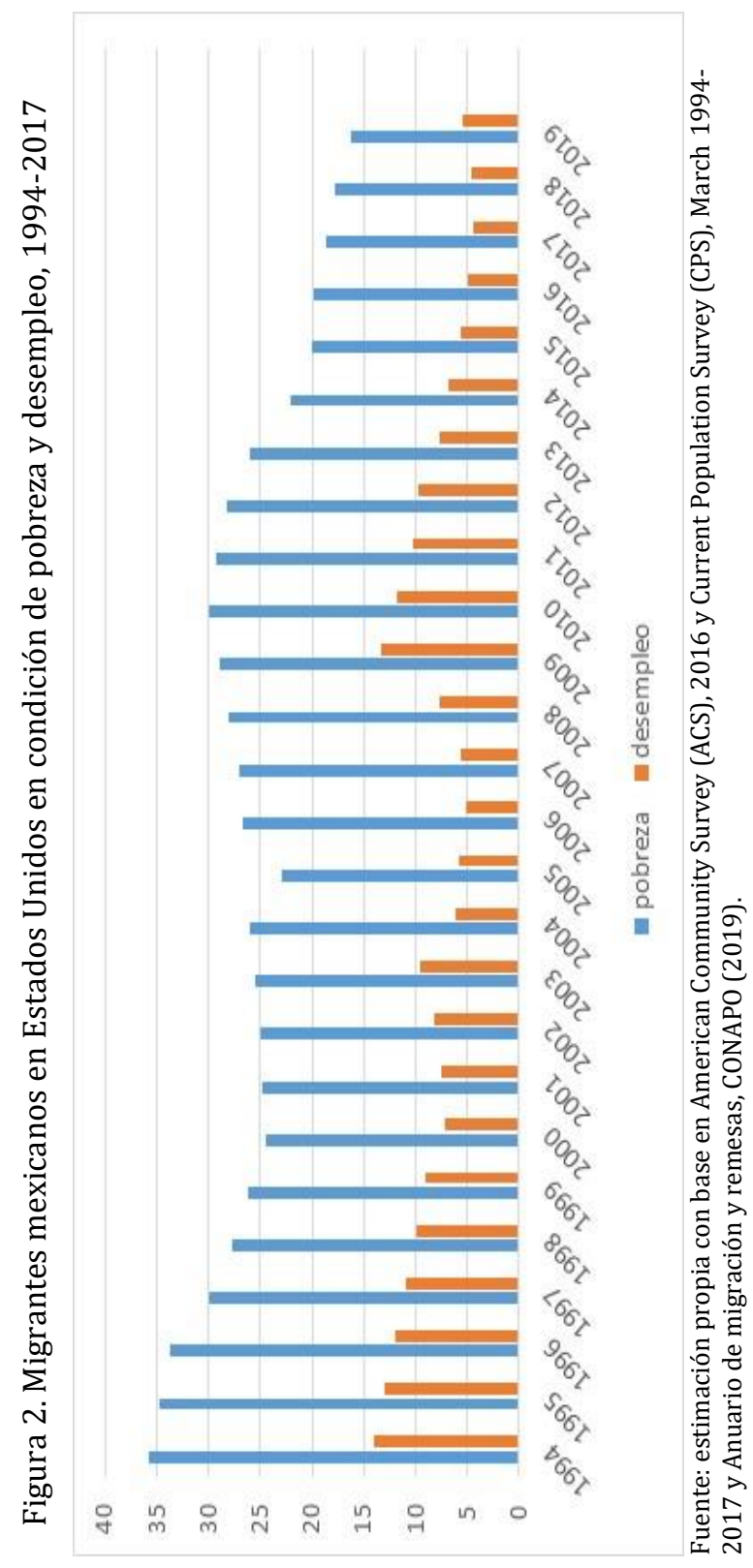


El Coronavirus colocó una dinámica comportamental individualista, desapegada de los hilos asociativos, marcada por el consumo y la competencia, lejos de todo armado comunitario, incluso familiares, las tramas de socialización bastante deterioradas, sin habilidades, ni conocimientos para vivir o afrontar una nube intensa de incertidumbre, zozobra o angustia ante la amenaza de nuestros bienes, el riesgo y desventura para defender la familia y nuestra propia vida (Salazar, 2020: 203).

En medio de este negro panorama, que se suscita a nivel mundial, los migrantes mexicanos en Estados Unidos, pese a enfrentar condiciones particularmente difíciles por la recesión económica, el incremento de la hostilidad gubernamental y los peligros que entraña la pandemia, han seguido enviando dinero a sus familias en México y esto se explica en torno a lo que sostiene Canales (2005: 170), que las remesas "reproducen relaciones de reciprocidad, responsabilidad y solidaridad, mismas que resultan fundamentales en la conformación de todo sistema familiar extenso" y que en momentos de apremio son cuando más se presentan en un clima adverso como la presente pandemia. Más todavía, de acuerdo con el Banco de México (2020), las remesas no sólo no disminuyeron, un poco más de 4,016 millones de dólares en marzo de 2020, lo que significó 35 por ciento de aumento respecto a marzo de 2019 y 18 por ciento más con relación a marzo de 2020 , como se ve en la Figura 3. Según el informe del Banco de México (2020), el número total de transacciones aumentó 15.3 por ciento interanual a 10.6 millones, mientras que el monto promedio por envío se elevó 18.1 por ciento de 378 dólares, donde el monto promedio es de 300 (Forbes, 2020b). En este año de pandemia, la cifra para junio volvió a incrementarse en 2,861 millones de dólares, lo que implicó el segundo monto más alto enviado para un mes similar desde que se tiene registro (1995), luego del reportado en abril de 2019 de 2,937 millones de dólares, por lo que implicó un descenso anual de 2.6 por ciento, aunque bajaron en nueve dólares, en este bimestre de mayo a junio en el promedio de envío, esto se vio ejemplificado por un número superior de envíos.

Esta situación no es ajena de los migrantes mexicanos en el envío de remesas, ante los problemas económicos que padece México, por ejemplo, en 2015, por primera vez, las remesas familiares superaron a las exportaciones petroleras (Pintor y Rocha 2018). 
El impacto económico de las remesas en el ingreso de las familias ...

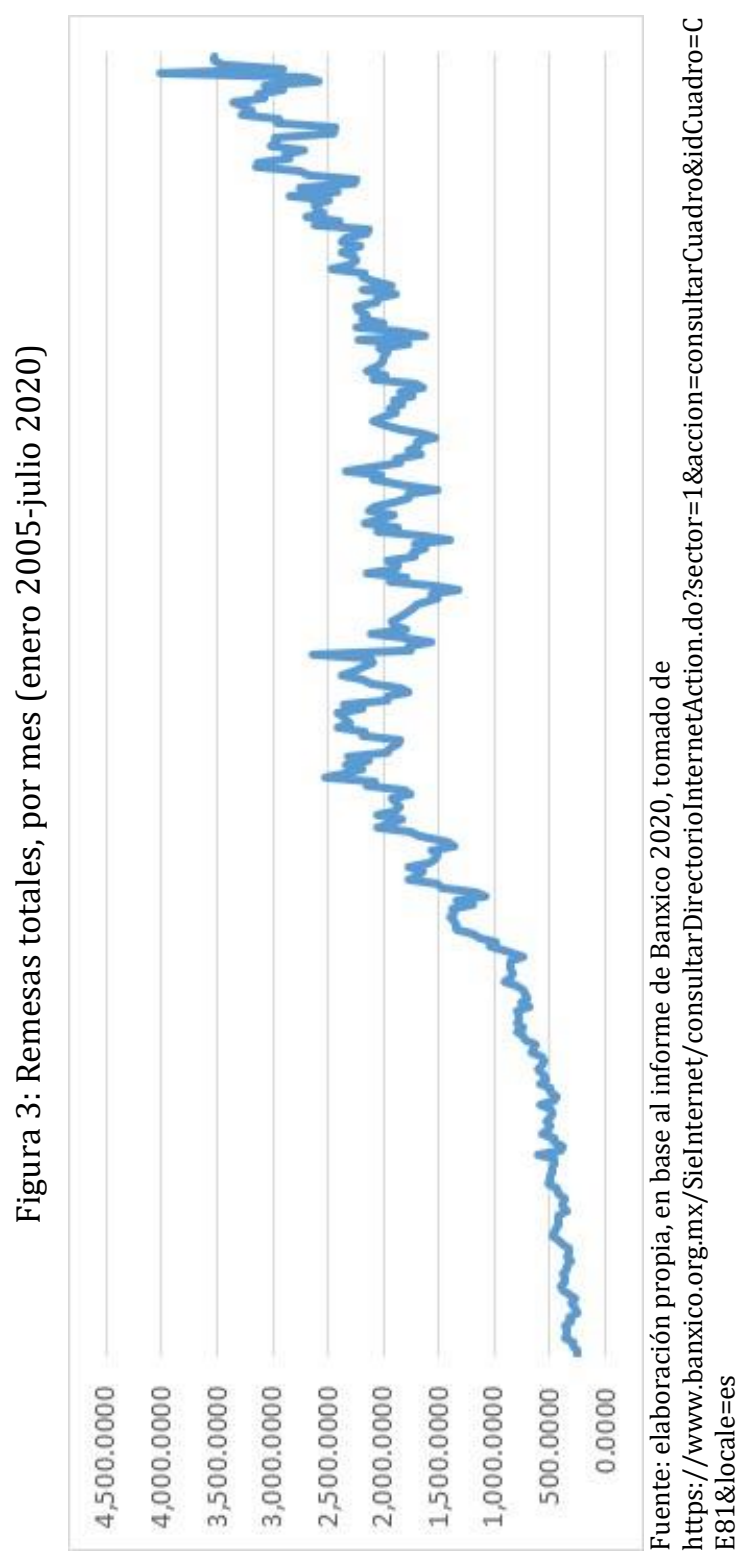

Año 5 No. 10 julio-diciembre 2020 
Estos recursos de los migrantes resultaron algo inédito en la economía mexicana donde alrededor de 30 por ciento del presupuesto del gobierno proviene de las exportaciones de petróleo.

Las remesas en ese año alcanzaron en esos cinco primeros meses la cantidad de 7735 millones de dólares, mientras que Pemex vendió en petróleo la cantidad de 6,678 millones de dólares (Pintor y Rocha, 2018).

Otro de los pasajes recientes de la historia de los migra dólares, fue lo sucedido en el 2018, donde la economía mexicana se contrajo, al tener un crecimiento entre 0.1 y 0.4 por ciento. La respuesta del presidente de la República, Andrés Manuel López Obrador, durante el primer informe del presidente de México, se pronunciaba:

Hay que desechar la obsesión tecnocrática de medir todo con el crecimiento económico, considerando que lo fundamental no es lo cuantitativo sino la distribución equitativa de la riqueza y el fin último de un buen gobierno es conseguir la felicidad de la gente. El crecimiento económico y los incrementos en la productividad no tienen objetivos en sí mismos, sino como medios para lograr un objetivo superior, el bienestar general de la población (Morales, 2019).

Las palabras del presidente de México versaban sobre el hecho del pobre desempeño en materia de crecimiento, pero a su vez, señalaba lo importante que son las remesas familiares en México, como sinónimo de "solidaridad de paisanos", dado que, en los primeros seis meses de 2018, se recibieron remesas por 16,845 millones de dólares, el monto más alto que se había registrado en toda la historia del país. Aunque, las remesas familiares, posteriormente, durante 2019, volvieron a su ritmo natural, hasta que llega el conflicto de la pandemia, donde nuevamente cobran importancia, dado los lazos que mantienen los migrantes con sus familias y comunidades de origen.

Sobre esto cabe preguntarse dos situaciones, la primera: $i$ a qué se debe el aumento de las remesas en los últimos lustros?, o bien, ¿Qué tipo de estrategias emplean los migrantes mexicanos para mejorar los ingresos de las familias migrantes, incluso ante el temor de la deportación y el costo de migrar nuevamente? Se ha detectado que, en varios periodos de la historia de la migración de mexicanos a Estados Unidos, cuando incrementa la política de deportaciones y los sentimientos antiinmigrantes en Estados Unidos, existe un crecimiento de remesas hacia México (Pintor y Rocha, 2020). 
Si bien, en la reciente historia de las deportaciones de mexicanos de Estados Unidos, que han ocurrido, como nos dice Meza (2014) o Peña (2015), donde las deportaciones han crecido 16 por ciento mensuales (42 por ciento anual, entre 2017 y 2018, siendo de 2018 a 2019, que en promedio rondó los 18 mil casos al mes. 0 bien, el temor de la comunidad emigrada mexicana en Estados Unidos, lo constituye el hecho de poner gravamen a las remesas de dos por ciento, sobre el envío del monto total. Cabe señalar que Oklahoma cobra un impuesto a las remesas de cinco dólares a los primeros 500 y uno por ciento a los montos superiores, bajo la ley de ese estado titulada HB-2250, propuesta el 1 de julio del 2009, pero aceptada el 30 de junio de 2016. Otros dos estados, Iowa y Georgia, están analizando un gravamen más amplio que incluiría no solo a las remesas sino también otros tipos de transferencias (Cuevas-Mohr, 2016).

Entre las respuestas que pueden darse ante el incremento de las remesas, en estos últimos periodos y más ante el problema de la pandemia global, tienen que ver con la naturaleza misma de la remesa, no sólo como recurso económico, sino como parte de una estrategia que va desde lo familiar e individual. 0 bien, como antepone Moctezuma (2011: 31): "las remesas familiares expresan un conjunto de relaciones sociales y afectivas que es necesario develar y explicar, según sean sus contextos", donde más allá que son recursos monetarios que los emigrantes obtienen trabajando en el extranjero y luego los envían a su país natal, son una de las consecuencias más visibles de la emigración en que ésta se origina.

Si bien, las remesas forman parte del ingreso corriente del gasto de los hogares, no siempre funcionan de la misma forma, por ejemplo, los trabajos realizados por López et al. (2011) Díaz y Ramírez (2017) y Pintor et al. (2017) anteponen que existen aparte el uso de las remesas como gasto específico, donde estas tienden a incrementarse en ciertos periodos, como el día de las madres o la navidad; celebraciones como los XV años, graduaciones o cumpleaños; pero que también estas pueden utilizarse ante problemas suscitados al margen del uso corriente, como la enfermedad, deudas u otros gastos, donde independientemente de lo instrumental del objeto, encierran expresiones de solidaridad en la modalidad de subsidios a la economía familiar.

Ambas remesas, tanto "específicas" como las que funcionan como "gasto corriente", son un recurso que más allá de la mera necesidad económica, son expresiones que se interiorizan distintas maneras 
culturales e ideológicas particulares, los valores cambiantes y expectativas afloran asimismo en la profundidad y expansión del proceso migratorio (Pintor et al., 2017).

Es así, como se puede observar en el Mapa 2, donde las regiones, llamadas tradicionales de la migración mexicana, no hayan bajado su recepción de remesas, tal y como lo demuestran los datos, se estima que las remesas en México equivalen a 2.8 por ciento de dependencia del PIB, mientas que por entidades federativas, por ejemplo, los registros marcan que los estados que reciben más remesas son Jalisco (10.5), Michoacán (10.3) Oaxaca (5.2), Puebla (4.7), entre otros (como se observa en el Mapa 2); pero si lo comparamos con el número de hogares por dependencia de las remesas (porcentaje de las remesas con respecto al PIB), las entidades líderes son: Michoacán (11.4 por ciento), Oaxaca (10.1 por ciento) y Zacatecas (7.9 por ciento).

Mapa 2. Remesas recibidas en los estados (millones de dólares, enero-marzo 2017)

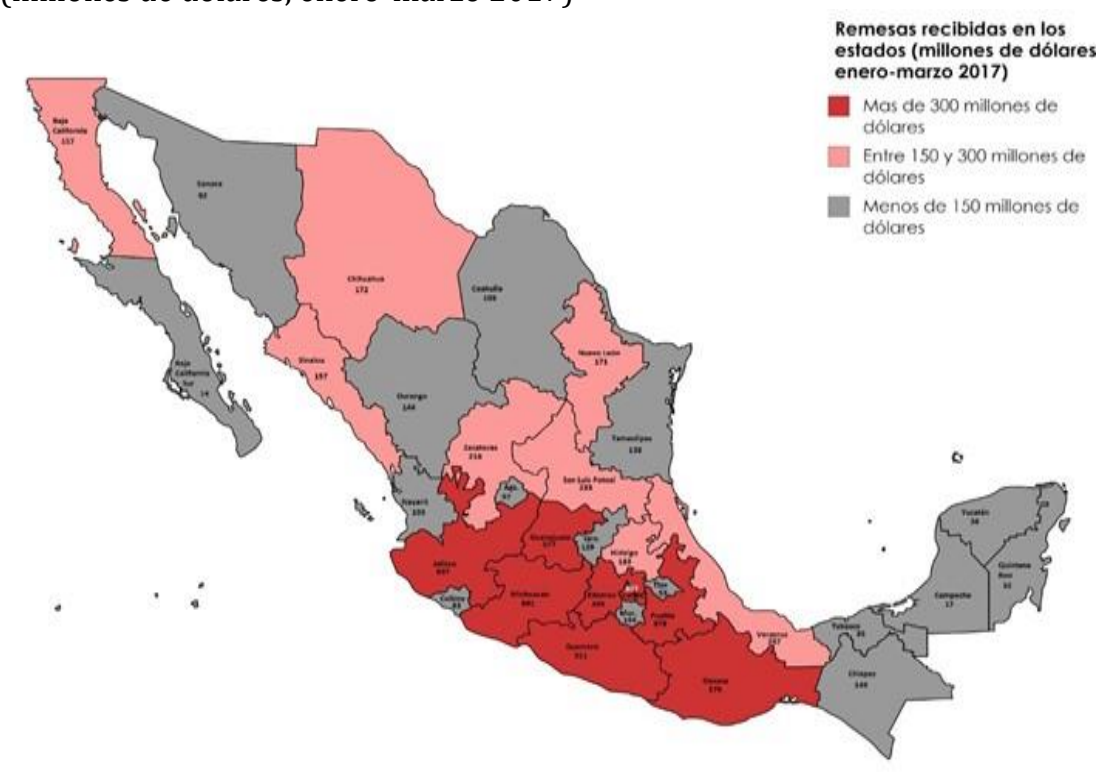

Fuente: elaboración propia, en base a los datos del INEGI (Tasa de emigración, ENOE), Banxico (2020), Remesas.

En cuanto a los municipios, la tasa más alta la tiene, Dolores Hidalgo, que registra dos de cada diez viviendas receptoras de ingresos vía 
remesas, teniendo el porcentaje más elevado de México con 19.6 por ciento, le siguen Salvatierra 18.9 por ciento; Pénjamo, 18.7 por ciento; San Felipe, 18.4 por ciento, Acámbaro, 18.2 por ciento y Valle de Santiago con 14.7 por ciento. Los porcentajes más altos a nivel nacional indican que siete de diez, son municipios de Guanajuato, completando la lista Apatzingán 16.43 por ciento, La Piedad 13.7 e Hidalgo (Michoacán) junto con Atlixco (Puebla) con 13 por ciento, como los hogares que reciben más remesas, en nociones de consumo y gasto, vía remesas. En cuanto al volumen total, la ciudad de Tijuana, Baja California, acumuló un monto de 102.6 millones de dólares en el periodo de enero a marzo de 2019, siguiendo Puebla, con 98.2 millones. En la lista de los diez municipios con mayor ingreso de dólares también aparecen: Morelia, Michoacán, con 94.4 millones de dólares, Guadalajara, Jalisco con 91.6 millones y Culiacán, Sinaloa con 81.9 millones de dólares. Seguidos de: Zapopan, Jalisco y León, Guanajuato con 71.3 millones de dólares, cada uno, Oaxaca con 70.7 millones, Álvaro Obregón, en Ciudad de México con 70.6 millones y Juárez, Chihuahua, con 66.9 millones de dólares (BBVA Research, 2020).

En suma, es importante replantear el significado e importancia, tanto macro como micro, sobre el discurso y los estudios de las remesas familiares, su relevancia de estas radica en que las familias se ven beneficiadas con el uso de ellas, ya que representan en las comunidades donde se reciben, cambios económicos debido al uso productivo de las mismas, mientras que en la mayoría significan un ingreso más, en muchos de los casos, son el único sustento familiar o el ingreso más representativo para el consumo, donde generalmente se da en las comunidades más o menos pobladas.

En lo referente a las remesas y al problema de la pandemia, su comportamiento cíclico e inestable, podrán modificar su alza relativamente, como lo apunta el laboratorio Regional de la Universidad Nacional Autónoma de México (UNAM) (Valdivia et al., 2020) que prevén que existirá una disminución anual de las remesas en términos reales y en pesos, entre 17 y menos de 20 por ciento, con relación a 2019, sus estimaciones indican que ocurrirá una caída, de 36 mil millones de dólares que alcanzó en 2019, a cerca de 28,800 millones en 2020 (Valdivia et al., 2020). Esta misma investigación, arroja que los estados que tendrán mayor capacidad para suavizar la caída del empleo y el ingreso durante la pandemia serán Aguascalientes, Morelos, Oaxaca y Sinaloa, y es muy probable que se sumen los estados de Guanajuato, Hidalgo 
y Tlaxcala (Valdivia et al., 2020). Mientras que los estados que se estiman como focos rojos, donde las remesas no lograrán contrarrestar los efectos negativos de la crisis económica son: Puebla, San Luis Potosí y Nayarit. Por último, las entidades federativas que perderán su capacidad contra cíclica durante la pandemia son Zacatecas, Durango, Guerrero, Jalisco, Michoacán y Chiapas. Estados que conforman, en su mayoría, la llamada región histórica de la migración mexicana (Valdivia et al., 2020),

De una manera general, durante la pandemia, se puede entender que estos flujos económicos han encontrado distintas aristas, por ejemplo, 19 entidades federativas presentaron crecimientos en su flujo de remesas y 13 estados contracciones. Baja California tuvo un repunte de 36.9 por ciento en la recepción de remesas, mientras que otros estados con importantes crecimientos durante este periodo fueron: Sonora $(+17.3$ por ciento), Sinaloa $(+16.7$ por ciento), Chihuahua $(+15.5$ por ciento) y Jalisco $(+14.6$ por ciento). Los mayores decrementos se observaron en Tabasco (-29.6 por ciento), Yucatán (-19.9 por ciento) y Tlaxcala (-19.4 por ciento) (Índice Corporativo, 2020).

Sobre estos datos, podemos entender que gran parte de las remesas familiares durante la pandemia se hicieron por migrantes transfronterizos o los llamados commuters, siendo los estados del Noroccidente y del Noroeste, donde personas que debido a la restricción fronteriza realizaron depósitos electrónicos. Mientras que, en los estados llamados tradicionales, permitió que las viejas prácticas transnacionales, no se vieran tan afectadas por la crisis del Covid-19. Este mayor arraigo puede estar relacionado con un mayor porcentaje de migrantes con ciudadanía y residencia permanente, más empleos formales y de mayor calidad, y mayores redes sociales (amigos y familiares) de apoyo y protección.

Por último, las remesas de los migrantes de más reciente llegada y con menos redes sociales pudieron haber sido las más afectadas por la crisis por Covid-19. La mayoría de los estados en México que tuvieron contracciones en las remesas fueron de la región Centro y Sursureste. Gran parte de las diásporas de estas entidades en Estados Unidos se formaron en los últimos 30 años; mientras que en la región Tradicional y los estados fronterizos, tienen diásporas de más décadas. 


\section{CONCLuSiones}

Más allá de los análisis que se pueden verter sobre el monto de las remesas durante la pandemia, éstas significan que, para muchos hogares mexicanos son la principal fuente de subsistencia, localizadas en comunidades de escaso desarrollo regional. De igual forma, sus aportes constituyeron un eje estratégico y central en la balanza de pagos, siendo fundamental a nivel local y regional. Por lo que, a nivel nacional, su disminución podrá impactar en el Producto Interno Bruto, afectando en los niveles de pobreza de los segmentos más vulnerables de la población y que han tenido un impacto favorable sobre el bienestar de las familias.

Sin embargo, su forma y su contexto, como el ser cíclicas e inestables en el tiempo, pueden contradecir cualquier tipo de análisis que se pueden realizar de sus montos, ya que, no son recursos unitarios, sino que entran en una gama más amplia, al ser correlacionadas con el desarrollo de lo familiar. Es ahí que las remesas, puedan seguir al alza, en el sentido que su característica primordial, es hacer que la familia subsista. En pocas palabras, su centralidad, no es lo meramente económico, sino el impacto social que éstas han tenido en el desarrollo de sus familias y sus localidades de origen.

Aun cuando estos vínculos bidireccionales iniciales dependen de la decisión del migrante de enviar remesas a sus familiares a su localidad de origen, su condición indocumentada, nivel de ingreso, el mercado de trabajo, edad, sexo, dominio del idioma o región, esto se encuentra estrechamente ligada con la relación que guarda el migrante con el hogar receptor y las necesidades de la familia.

En sí, durante la pandemia global, su comportamiento será cíclico, como se apunta, pero que en algunos de los casos funcionarán bajo el esquema de remesas específicas, pero de igual forma, se verán casos que familias de migrantes radicados en México, manden dinero a sus familiares de origen (funcionamiento transnacional) con el objetivo de evitar el retorno o la deportación. La fortaleza que adquieren las remesas familiares es que a futuro garantiza el bien familiar. También los envíos dependerán en gran medida de los objetivos o planes a futuro del emigrante, si éste ya tiene familia en Estados Unidos, tal vez no piense en regresar y los envíos se reducen considerablemente.

Otro punto a considerar, sobre el apoyo que los migrantes realizarán ante esta pandemia, es que éstos recursos no serán suficientes 
para contrarrestar la situación estructural de desigualdad social y de desempleo que se vivirá en el país, ya que sus usos, serán el autoconsumo de bienes de primera necesidad, tales como alimento, vestido y servicios de vivienda, por lo que su ingreso, aún que se haya incrementado en los meses de marzo a julio de 2020, se asemeja a un sueldo agrícola nacional, donde una economía de lento crecimiento o negativo, premiará la actitud de los migrantes.

\section{REFERENCIAS BIBLIOGRÁFICAS}

Alarcón, R., Cruz, R., Díaz-Bautista, A., González, G., Izquierdo, A., Yrizar, G., y Zenteno, R. (2009). "La crisis financiera en Estados Unidos y su impacto en la migración mexicana", en Migraciones Internacionales, 5(1), 193-210.

Americadigital (2020). “¿Cuántos casos de coronavirus hay en Estados Unidos?". Disponible en https://www.americadigital.com/estados-unidos/ cuantos-casos-de-coronavirus-hay-en-estados-unidos-45930

Armendares, P. (2018). La política migratoria de Trump. Impactos para los migrantes mexicanos y sus comunidades. México: Senado de la República.

Arroyo, J. y Berumen, S (2000). "Efectos subregionales de las remesas de emigrantes mexicanos en Estados Unidos", en Comercio Exterior, México, 50 (4). 340-349.

BBVA-Research (2018). Voto de los mexicanos en el exterior. Disponible en https://www.bbvaresearch.com/wpcontent/uploads/2018/07/VotoMexicanosdelExterior_20180716.pdf

BBVA-Research (2020). Análisis regional de las remesas en México. Disponible en https://www.bbvaresearch.com/publicaciones/mexico-hay1-65-millones-de-hogares-que-reciben-remesas-4-7-del-total/

Binford, L (2003). "Migrant and (Under) Development in México", en Critique and Anthropology, 23(3). 305-336

Borbón, R. (2020). La crisis de la globalización Reflexiones sobre la primera pandemia de la historia en el siglo XXI. México: Creativos7editorial.

Bureau of Labour of Statistics (BLS) (2020). The Employment Situation, april 2020. 3 de mayo. Disponible en https://www.bls.gov/news.release/ archives/empsit_05082020.pdf

Calderón, C. (2015). "La Inversión Extranjera Directa y las Remesas en México", en Cultura Científica y Tecnológica, 8(42), 32-42.

Canales, A. (2005). "El papel de las remesas en la configuración de relaciones familiares transnacionales", en Papeles de Población, 44, 149-172.

Canales, A. (2015). "El Debate sobre Migración y Desarrollo. Evidencias y aportes desde América Latina”, en Latin American Resarch Review, 50(1), 29-53. 
Carling, J. (2007). "Interrogar a las remesas: preguntas centrales para reflexiones más profundas y políticas más adecuadas", en Castles, S. \& Delgado Wise, R. (coords.), Migración y desarrollo: perspectivas desde el sur (pp. 51-73). México: Miguel Ángel Porrúa. https://doi.org/10.13140/ RG.2.1.4919.5126

Carvajal, L. y Almonte, L. (2011). "Remesas y crecimiento: un análisis estructural para México", en Análisis Económico, 26(62), 209-228.

Castles, S. (2016). "Understanding global migration: a social transformation perspective", in Amelina, A., Horvath, K. y Meeus, B. (eds.). An Anthology of Migration and Social Transformation (pp. 19-41). Switzerland: Springer.

Celorio, M. (2020). “Op-Ed: California's farm workers desperately need PPE and coronavirus tests", in Los Angeles Times, 14 de abril. Disponible en https://www.latimes.com/opinion/story/2020-04-14/coronavirus-farm-workers-testing

Clemente, Y., Grasso, D., Blanco, P., Sevillano, L. y Zafra, M. (2021). “Casos confirmados de coronavirus en España y en el mundo", en El País, 12 de febrero. Disponible en https://elpais.com/sociedad/2020/07/27/actualidad/1595838623_808240.html

CNN en español (2020). "La economía de Estados Unidos pierde un récord de 20,5 millones de empleos en abril", en CNN En Español, 8 de mayo. Disponible en https://cnnespanol.cnn.com/2020/05/08/la-economia-deestados-unidos-pierde-un-record-de-205-millones-de-empleos-en-abril/

CONAPO (2017). Anuario de migración y Remesas. México: Consejo Nacional de Población (CONAPO).

CONAPO (2019). Anuario de migración y Remesas. México: Consejo Nacional de Población (CONAPO).

Conway, D y Cohen, J. (1998). “Consequences of Migration and Remittances for Mexican Transnational Communities", in Economic Geography, 74(1): 26-44.

Cueto, J., García Castro, I. y Burgueño, N. (2019). “Retorno actual como estrategia resiliente de sobrevivencia de familias mexicanas establecidas en Arizona", en NuestrAmérica, 7(13), 241-263.

Cuevas-Mohr, H. (2016). The Taxing of Remittances in the US. Disponible en https://imtconferences.com/taxingremittancesus $/$ ? utm_source $=$ IMTC\% 27 s + Newsletters \&utm_campaig $\mathrm{n}=3 \mathrm{c} 70349465 \mathrm{EMAIL} \_C A M P A I G N \_2017 \_03 \_07 \& u$ tm_medium=email\&utm_term=0_082dfaaec4-3c70349465-281771489\&goal=0_082dfaaec4-3c70349465-281771489

Chishti, M. y Hipsman, F. (2015). "In historic shift, new migration flows from Mexico fall below those from China and India”. Disponible en ht- 
tps://www.migrationpolicy.org/article/historic-shift-new-migrationflows-mexico-fall-below-those-china-and-india

Diario Farma (2020). "Enfermedad por coronavirus, Covid-19: información científico-técnica. 23 de marzo de 2020", en Diario Farma, 27 de marzo. Disponible en https://www.diariofarma.com/2020/03/27/enfermedad-por-coronavirus-covid-19-informacion-cientifico-tecnica

Díaz, E. y Ramírez, J. (2017). "Gastos catastróficos en salud, transferencias gubernamentales y remesas en México", en Papeles de población, 23(91), 65-91. http://dx.doi.org/10.22185/24487147.2017.91.004

Espinel, M. (2020). "Geografía política de los cuidados (0 por qué la pandemia del coronavirus confinó a buena parte del Norte global)", en Geopolítica(s), 11, 127-140. https://doi.org/10.5209/geop.69252

Forbes (2020a). "Pandemia de coronavirus deja sin empleo a 22 millones en Estados Unidos", en Forbes. Disponible en https://www.forbes.com. $\mathrm{mx}$ /mundo-pandemia-coronavirus-22-millones-sin-empleo-eu/

Forbes (2020b). "Remesas a México sorprenden con récord en marzo pese a coronavirus", en Forbes. Disponible en https://www.forbes.com.mx/ economia-remesas-mexico-marzo-record-pese coronavirus/?utm_sour$c e=$ norapush\&utm_medium=pushnotifications\&utm_campaign=new-nora-push

González, R. (2015). “Las Remesas de EU superaron el ingreso por exportación petrolera", en La Jornada, 2 de septiembre. Disponible en https:// www.jornada.com.mx/2015/09/02/economia/025n1eco

Índice Corporativo (2020). "Remesas en tiempo de Covid-19", en Índice Corporativo, 4 de agosto. Disponible en https://indicecorporativo. com/2020/08/04/remesas-en-tiempos-de-covid-19/

Instituto de los Mexicanos en el Exterior (IME) 2018). Mexicanos en Estados Unidos - Datos, gráficos y mapas (Cifras 2017 y 2018). Disponible en https://www.gob.mx/conapo/articulos/mexicanos-en-estados-unidosdatos-graficos-y-mapas-cifras-2017-y-2018?idiom=es

Liu, Yuan; Ning, Zhi; Chen Yu et al. (2020). "Aerodynamic Characteristics and RNA Concentration of SARS-CoV-2 Aerosol in Wuhan Hospitals during COVID-19 Outbreak", in BioRxiv. Doi: https://doi. org/10.1101/2020.03.08.982637

López, J., Sovilla, B. y García, F. (2011). "Efectos macroeconómicos de las remesas en la economía mexicana y de Chiapas", en Papeles de población, 17(67), 57-89.

Loyer, B. y Giblin, B. (2020). “Qué mundo geopolítico después de 2020?”, en Geopolítica(s), 11, 115-126. https://doi.org/10.5209/geop.69250

Magaña, L. (2013). “Arizona's immigration policies SB1070", in Magaña, L. y Lee, E. (eds.). Latino politics and Arizona immigration law SB1070. USA: Springer. 
El impacto económico de las remesas en el ingreso de las familias ...

Manjarrez, J. (2020). "Las mujeres migrantes y el Covid-19”. Observatorio de Violencia de Género en Medios de Comunicación, 19 de mayo. Disponible en https://ovigem.org/las-mujeres-migrantes-y-la-pandemia-de-covid-19/05/2020/

Mazurek, J. y Girija, S. (2018). "Prevalence of Asthma, Asthma Attacks, and Emergency Department Visits for Asthma Among Working Adults - National Health Interview Survey, 2011-2016", en MMWR Morb Mortal Wkly. Rep., 67(13), 377-386. https://doi.org/10.15585/mmwr.mm6713a1

Meza, L. (2014). "Mexicanos deportados desde Estados Unidos: Análisis desde las cifras", en Migraciones Internacionales, 7(4), 265-276.

Moctezuma, M. (2011). La transnacionalidad de los sujetos. México: Porrúa

Moctezuma-Longoria, M., Becerril, J., y Piñeiro, R. (2018). “La migración transnacional entre México-Estados Unidos: un acercamiento sociodemográfico, 2014", en Huellas de la Migración, 3(5), pp.11-41. Disponible en de https://huellasdelamigracion.uaemex.mx/article/view/10883_

Montoya, E. (2004). Exportando trabajo. Importando Progreso. Migración mexicana a EU y remesas en Gabriel Leyva Solano: México: DIFOCUR-UAS.

Morales, Y. (2019). "Remesas, determinante aportación a la fortaleza económica durante primer año de gobierno: AMLOE", en El Economista, 2 de septiembre. Disponible en https://www.eleconomista.com.mx/economia/Remesas-determinante-aportacion-a-la-fortaleza-economica-durante-primer-ano-de-gobierno-AMLO-20190902-0023.html

OCDE (2018). Indicators of Immigrant Integration: Settling In. Francia: OCDE. Disponible en https://www.oecd-ilibrary.org/docserver/ 9789264307216-en.pdf?expires $=1589225240 \& i d=i d \& a c c n a m e=$ gues t\&checksum=BD9D37647C31C08702DFCF8B724D54B2

OIM (2020). Informe sobre las migraciones en el mundo 2020. Suiza: ONU-Migración.

OMS (2020). Report of the WHO-China Joint Mission on Coronavirus Disease 2019 (Covid-19). Disponible en https://www.who.int/docs/default-source/coronaviruse/who-china-joint-mission-oncovid-19-finalreport.pdf

Peña, J. (2015). "Perfil laboral de migrantes mexicanos deportados e inserción laboral en México”, en Migración y Desarrollo, 13(24), 167-184.

Pintor, R. y Rocha, D. (2018). "Los migradólares en la era de Trump. ¿Cuánto impactarán en México?", en Munguía, A. (coord.). Los retos de la migración en el siglo XXI. México: BUAP.

Pintor, R. y Rocha, D. (2020). "Acciones y discursos de las remesas en el panorama Binacional", en Rodríguez, J. y Valdés, G. (coord). Impacto de la política migrante en niños, niñas, adolescentes migrantes: origen, tránsito y retorno. Enfoque regional fronterizo. México: COLSON- UNISON. 
Pintor, R., Peraza, B. y Heredia, K. (2017). “Impacto de las remesas familiares y el programa "Prospera" en jóvenes de nivel medio superior en Choix, Sinaloa, México", en Ánfora, 24(42), 19-43. https://doi.org/10.30854/anf. v24.n42.2017.165

Robertson, S. (2018). "Status-making: Rethinking migrant categories", in Journal of Sociology, 55(2), 219-233. https://doi. org/10.1177/1440783318791761

Salazar, R. (2020). "Pandemia y disciplinamiento social, autoencierro con miedos y controles de obediencia”, en Conjeturas Sociológicas, 8(21), 230249.

Serrano, C. (2020). "Coronavirus en EE.UU. El devastador impacto del covid-19 entre los afroamericanos", in $B B C$. Disponible en https://www.bbc. com/mundo/noticias-internacional-52219474

Suro, Roberto (2005). "Las remesas y el riesgo", en Terry, D. y Wilson, S. (coords.), Remesas de Inmigrantes: Moneda de cambio económico y social (3-31). Washington: BID.

USCB (2018). Current Population Survey. Disponible en https://www.census.gov/programs-surveys/cps.html

Valdivia, M. (2020). "Remesas y Coronavirus. El caso de México", en Nexos. Disponible en https://migracion.nexos.com.mx/2020/04/remesas-y-coronavirus-el-caso-de mexico/?fbclid=IwAR2hxPbhsert9pf9pBWH6Un2FEWQn_2XKRhG9EhpNoirFR3zI7mWRbd3lRA

Valdivia, M., Quintana, L., Mendoza, M. y Salas, C. (2020). Informe 2. Impacto del COVID-19 sobre el envío de remesas a México y sus efectos en las economías regionales. México: UNAM

Zong, J., Batalova, J. y Burrows, M. (2019). “Frequently Requested Statistics on Immigrants and Immigration in the United States. Migration Policy Institute". Disponible en https://www.migrationpolicy.org/article/frequently-requested-statistics-immigrants-and-immigration-united-states-2020

\section{RESUMEN CURRICULAR DE LOS AUTORES}

\section{Renato Pintor Sandoval}

Doctor en Ciencias Políticas y Sociales con orientación en Relaciones Internacionales, por la Universidad Nacional Autónoma de México; Profesor-investigador de Tiempo Completo, titular C, en la Facultad de Estudios Internacionales y Políticas Públicas de la Universidad Autónoma de Sinaloa; con postdoctorado en la Universidad Autónoma de Baja California en la Facultad de Economía y Relaciones Internacionales, Tijuana; Miembro del Sistema Nacional de Investigadores Nivel I; miembro del Cuerpo Académico Consolidado: Redes Sociales y Cons- 
trucción del Espacio Público, Clave PRODEP: UAS-CA-257. Línea de investigación: Migración y Desarrollo; Transnacionalismo; Frontera y segmentación de mercados. Coordinador General de Posgrado de la FEIyPP. Miembro de la Red Iberoamericana de Academias de Investigación sobre Migración y Desarrollo CONACYT.

Dirección electrónica: renato_azul@hotmail.com; rpintor@uas.edu.mx Registro ORCID: http://orcid.org/0000-0003-3634-5011

Jesús Bojórquez Luque

Doctor en Historia por la Universidad Autónoma de Sinaloa (UAS); Maestro en Economía del Medio Ambiente y de los Recursos Naturales por la Universidad Autónoma de Baja California Sur (UABCS); Sociólogo por la UAS. Profesor-Investigador de la UABCS, miembro del Sistema Nacional de Investigadores Nivel C. Dentro de las últimas publicaciones se encuentran: Bojórquez, J. (2021). "Tierra de propiedad social, turismo y expansión urbana en San José del Cabo, Baja California Sur (México)". Pasos, 19(1), 85-99; Bojórquez, J. y Frías, E. (2020). "Primeros intentos de planeación urbana en Cabo San Lucas, Baja California Sur, México. Un análisis histórico". Revista Geográfica Venezolana, 61(2), 496-512; Bojórquez, J., Ángeles M. y Gámez, A. (2020). Videovigilancia y segregación espacial en tiempos del neoliberalismo autoritario. El caso de Los Cabos, Baja California Sur (México). CS, 31, 217-242.

Dirección electrónica: bojorquez@uabcs.mx

Registro ORCID: https://orcid.org/0000-0002-1745-4979 